\title{
Long term results after autologous nasal mucosal transplantation in severe mucus deficiency syndromes
}

\author{
Hartmut Wenkel, Volker Rummelt, Gottfried O H Naumann
}

\begin{abstract}
Aim-Severe mucus deficiency syndromes may require substitution of mucous membrane for re-establishment of the ocular surfaces. The long term results after autologous nasal mucosal transplantation were investigated.

Methods-55 eyes of 50 patients with severe mucus deficiency syndromes were followed retrospectively after free autologous nasal mucosal transplantationgroup A: patients after severe lye, acid, heat burns, or radiation $(n=38$ eyes), group B: patients with systemic mucosal disease ( $n=17$ eyes). The results of routine clinical examination were recorded and patients were followed for a median of 37 months. 17 biopsies of transplanted nasal mucosa were studied by light microscopy and 22 patients by impression cytology before and at several intervals after mucosal transplantation.
\end{abstract}

Results-All nasal mucosal grafts healed well and no intraoperative complications occurred. During follow up 107 additional surgical procedures were performed including 16 lamellar and 21 penetrating keratoplasties. Subjective complaints improved in $44 / 47$ patients with preoperative symptoms. Best corrected visual acuity at the end of follow up was increased in 23 eyes, 10 eyes $(18.2 \%)$ reached a final visual acuity equal to or greater than $20 / 200$. Histopathologically, all $(n=17)$ biopsies showed vital intraepithelial mucin producing goblet cells in the nasal mucosal graft (median 25 cells/field (400× magnification)). The mean density of goblet cells before transplantation was $48 / \mathrm{mm}^{2}$ and after nasal mucosal grafting $432 / \mathrm{mm}^{2}$ measured by impression cytology $(\mathrm{p}<0.0001)$.

Conclusions-Functional goblet cells persist in autologous nasal mucosa for up to 10 years after transplantation. In patients with severe mucus deficiency syndromes of different origin nasal mucosal transplantation can re-establish the ocular surface, substitute the mucus components of the tear film, improve symptoms of the patients, and facilitate a moderate increase in visual acuity.

(Br f Ophthalmol 2000;84:279-284)

Hartmut Wenkel, MD

Department of

Ophthalmology, University

of Erlangen-Nürnberg,

Schwabachanlage 6,

D-91054 Erlangen, Germany

Accepted for publication 8 November 1999
Lye, acid, and heat burns or systemic mucosal disease often lead to severe impairment of the conjunctiva with mucus deficiency. The de- struction of the normal conjunctiva frequently results in cicatrisation of the superficial ocular surfaces with firm symblepharon, pain, dry eye symptoms, and a decrease in visual acuity. Re-establishing the ocular surfaces and the normal tear film composition requires replacement of destroyed mucosa preferentially with tissue harbouring fully functional goblet cells capable of mucin production. Transplantation of autologous healthy conjunctiva of the fellow eye certainly represents the best treatment for these patients. ${ }^{1-3}$ However, in a majority of eye burns and all systemic mucosal diseases the fellow eye is not able to provide normal conjunctiva because of bilateral involvement of the disease. In severe ocular burns with involvement of the whole conjunctiva an additional problem may occur. The reconstruction of the fornix and the ocular surface may require more mucosa than one could safely excise from the fellow eye.

Various other mucosal tissues have been used to prevent formation of recurrent symblepharon and to reconstruct the ocular surfaces. ${ }^{4-8}$ Different authors have used buccal or lip mucosa, ${ }^{68}$ hard palate mucosa, ${ }^{9}$ maxillary sinus mucosa, ${ }^{10}$ or more recently amniotic membrane. ${ }^{11}{ }^{12}$ Over a decade ago we started using free autologous nasal mucosal grafts to improve the ocular surface and to relieve the symptoms of these patients. ${ }^{13}$ Nasal mucosa was used because of its richness in goblet cells not only in the subepithelial mucosal glands but also within the superficial epithelial cell layers (Fig 1). Further advantages are the easy accessibility and the great amount of nasal mucosa, which can be safely excised.

We now report the long term results after free autologous nasal mucosal grafting in 55 eyes of 50 patients with severe mucus deficiency syndromes. Patients with conjunctival impairment after trauma were analysed separately from patients with systemic mucosal disease to evaluate the potential benefit of the procedure in both groups of patients.

\section{Patients and methods}

Patients were examined retrospectively after autologous nasal mucosal transplantation performed at the department of ophthalmology in Erlangen, Germany, between 1984 and 1994. There were 55 eyes of 50 patients ( 39 men, 11 women) ranging in age from 5 to 83 years (median 43.5 years). Mucus deficiency was mainly due to severe lye (21 patients), acid (seven patients), and heat (six patients) burns. The other patients involved in the study suffered from radiation conjunctivopathy (two 


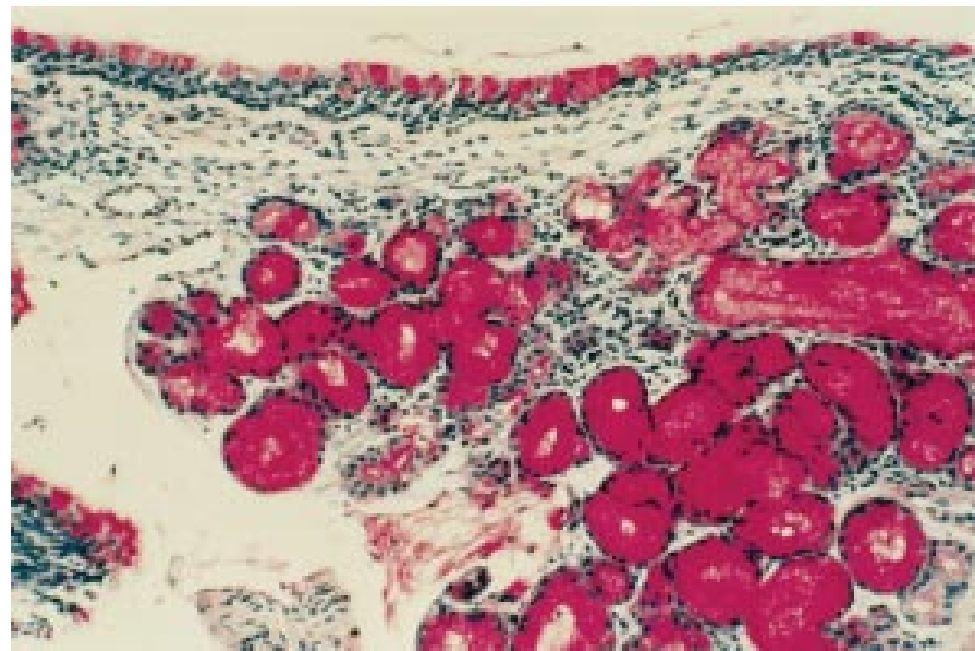

Figure 1 Autologous nasal mucosal transplant excised 8 years after transplantation with numerous epithelial and submucosal goblet cells. Thick nasal mucosa as a result of initially insufficient mucosal trimming before grafting (periodic acid-Schiff stain, original magnification $\times 100$ ).

patients), or systemic disease like FuchsStevens-Johnson syndrome (five patients), ocular pemphigoid (four patients), Lyell syndrome (four patients), or neurodermatitis (one patient).

Patients were subdivided into two groups for evaluation of the benefits of nasal mucosal transplantation. Group A consisted of patients with mucus deficiency after lye, acid, and thermal burns or as a result of local radiation (36 patients; 38 eyes). Group B consisted of patients with mucus deficiency syndromes following systemic diseases (14 patients; 17 eyes). In group $\mathrm{A}$ there were six female and 30 male patients ranging in age from 5 to 83 years (median 42.0 years). The interval between trauma and nasal mucosal grafting ranged from 4 days to 35 years (median 16 months). We included nine patients with nasal mucosal grafting within 14 days after ocular burns. First results of this early nasal mucosal grafting were very encouraging. ${ }^{14}$ In group B there were nine male and five female patients ranging in age from 5 to 72 years (median 50 years).

Almost all eyes treated showed severe mucus deficiency (52/55) with symblepharon (42/55) before transplantation (Fig 2A). Mucus deficiency was assessed clinically and was confirmed by impression cytology in 30 patients. Preoperative Schirmer's testing in 38 patients showed aqueous deficiency in $82 \%$ of patients. Three eyes in group A received mucosal trans-

Table 1 Clinical findings before nasal mucosal transplantation

\begin{tabular}{llll}
\hline Symptom & Eyes $(n=55)$ & Group A $(n=38)$ & Group B $(n=17)$ \\
\hline Visual decrease & $55(100 \%)$ & $38(100 \%)$ & $17(100 \%)$ \\
Mucus deficiency & $52(95 \%)$ & $35(92 \%)$ & $17(100 \%)$ \\
Vascular corneal scars & $43(78 \%)$ & $27(71 \%)$ & $16(94 \%)$ \\
Symblepharon & $42(76 \%)$ & $26(68 \%)$ & $16(94 \%)$ \\
Trichiasis & $23(42 \%)$ & $12(32 \%)$ & $11(65 \%)$ \\
Entropion & $11(20 \%)$ & $8(21 \%)$ & $3(18 \%)$ \\
Lagophthalmos & $11(20 \%)$ & $7(18 \%)$ & $4(24 \%)$ \\
Reduced ocular motility & $10(18 \%)$ & $6(16 \%)$ & $4(24 \%)$ \\
Endophthalmitis & $2(4 \%)$ & $2(5 \%)$ & - \\
& Patients $(n=50)$ & Group A $(n=37)$ & Group B $(n=14)$ \\
\hline Dry eye feeling & $45 / 50$ & $26 / 36$ & $14 / 14$ \\
Ocular pain & $33 / 50$ & $14 / 36$ & $9 / 14$ \\
\hline
\end{tabular}
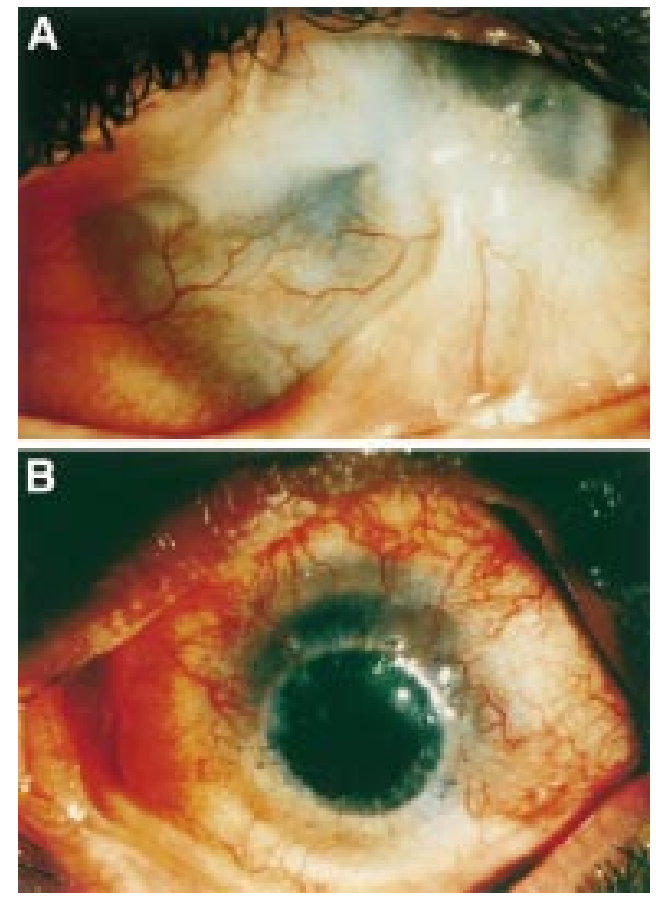

Figure 2 A 24 year old male patient with nitric acid burn to both eyes. (A) Preoperative: massive corneal

vascularisation and firm symblepharon in the left eye. (B) Postoperative: clear cornea and no symblepharon 5 months after penetrating keratoplasty and 8 months following autologous nasal mucosal transplantation.

plantation early after ocular burns and did not exhibit mucus deficiency at the time of transplantation. Preoperative clinical findings are listed in Table 1. Visual acuity of patients before transplantation ranged from light perception to $20 / 25$ vision with a median of counting fingers. Only seven patients had a visual acuity equal to or greater than $20 / 200$. Six of the latter patients belonged to group A. In 32 patients both eyes were involved (A: 18/36; B: 14/14). Patients were surveyed for a median of 37 months covering a range from 1 month to 10 years. The main clinical differences between groups A and B before surgery were a higher frequency of symblepharon $(p=$ 0.035 , Fisher's exact test) and dry eye ( $p=$ 0.016 , Fisher's exact test) in group B.

\section{METHODS}

Free autologous nasal mucosal transplantations were performed using a partial resection of the inferior or medial nasal turbinates as described in detail earlier. ${ }^{13}$ Briefly, all patients were examined carefully before transplantation surgery by an otorhinolaryngologist for the presence of sufficient vital nasal mucosa. Symblepharon was separated and necrotic or atrophic conjunctiva was completely resected. Following excision of nasal mucosa, adherent bone remnants or cavernous tissue were dissected and the tissue was trimmed into flat grafts. Transplants were fixed to the eye with single interrupted sutures from the limbus to the insertion of the rectus muscles and sutured to vascularised tissue in the fornix region. Transplant size varied according to the area requiring coverage. Samples of the prepared nasal mucosa and of the excised necrotic or 


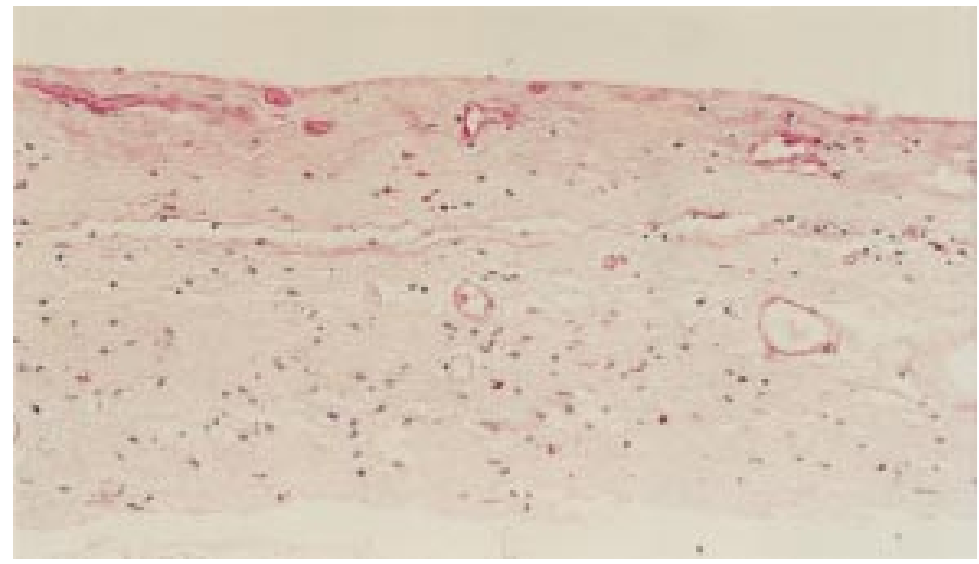

Figure 3 Histological appearance of surgically excised atrophic conjunctiva before nasal mucosal grafting 1 year after lye burn (periodic acid-Schiff stain, original magnification $\times 200)$

atrophic conjunctiva (Fig 3) were examined by routine histopathology in all cases. Clinical findings and subjective complaints of patients after transplantation were recorded. We were particularly interested in visual acuity, additional surgery, survival of corneal transplants, and possible complications like recurrent symblepharon or corneal neovascularisation. In 26 patients one presurgical and several postsurgical Schirmer tests (with anaesthesia) were performed. We also studied 17 biopsies of nasal mucosal grafts at different times after transplantation by light microscopy using haematoxylin and eosin and periodic acid-Schiff (PAS) staining. In 22 patients impression cytology before and at several time points after nasal mucosal transplantation (mean 4.6 years) was performed (Fig 4).

\section{Results}

NASAL MUCOSAL TRANSPLANTATION

In 18 patients only the right eye and in 27 patients only the left eye received nasal mucosal transplantation. In five patients mucosal grafting was performed in both eyes with an interval between operations of several months. In 27 eyes the transplanted tissue surrounded the cornea completely and in 28 eyes partially. In four eyes the entire cornea was covered by nasal mucosa. No complications occurred during surgery, neither during exci-

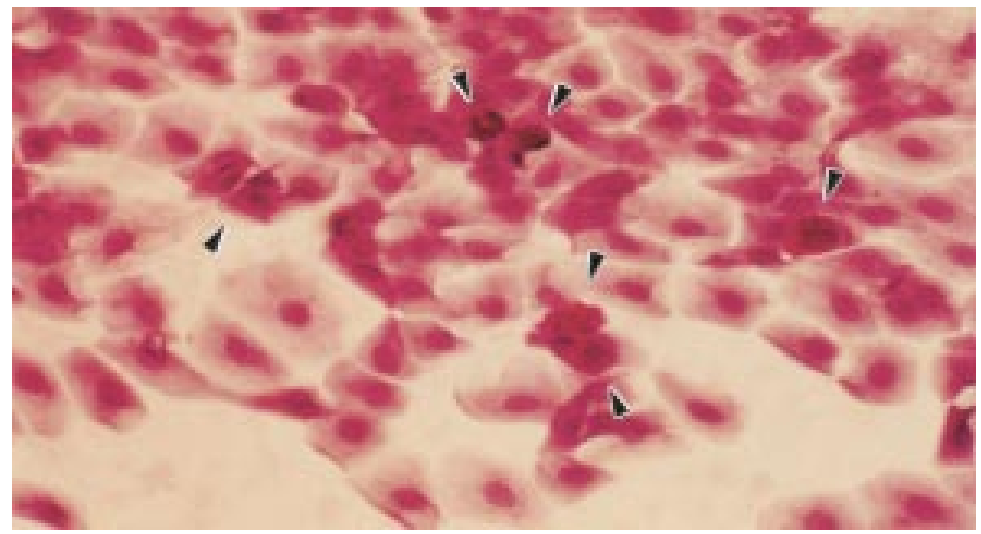

Figure 4 Impression cytology of the conjunctiva 6 years after autologous nasal mucosal grafting following severe lye burn, showing multiple goblet cells (arrowheads) (periodic acid-Schiff stain, original magnification $\times 400$ )
Table 2 Postsurgical findings and complications

\begin{tabular}{llll}
\hline Finding & Total & Group A & Group B \\
\hline Symblepharon & 13 & 6 & 7 \\
Trichiasis & 10 & 7 & 3 \\
Descemetocele & 5 & 3 & 2 \\
Glaucoma & 5 & 5 & - \\
Band keratopathy & 4 & 4 & - \\
Lagophthalmos & 4 & 3 & 1 \\
Endophthalmitis & 2 & 2 & - \\
Transplant melting & 2 & 1 & 1 \\
Corneal ulcer & 2 & 2 & - \\
Loss of anterior chamber & 2 & 2 & - \\
Macular hole & 1 & 1 & - \\
Hypotonia bulbi & 1 & 1 & - \\
Scleral necrosis & 1 & 1 & - \\
\hline
\end{tabular}

sion of the nasal mucosa nor during fixation to the globe (for example, inadvertent perforation of the globe).

POSTSURGICAL FINDINGS AND ADDITIONAL SURGERIES

Rapid healing of the grafts and establishment of an adequate episcleral blood supply were observed in all patients after transplantation. No necrosis of transplanted mucosa was noted in any of the patients. The fornix could be reconstructed in all eyes. There was no major aesthetic disfigurement in any patient. None of the patients suffered from any rhinological disorder - for example, atrophic rhinitis, after surgery. Postsurgically persisting or recurring symblepharon was seen in 13 eyes, however, without any major impairment of ocular motility. Seven of the latter patients belonged to group B (41.2\%) and six to group A (15.8\%) $(\mathrm{p}=0.047$, Fisher's exact test). A large number of additional postoperative findings (Table 2) were the result of intraocular complications after eye burns. During follow up additional operations were performed in 36 eyes (Table 3). In 27 eyes more than one surgical procedure was performed. Six patients received more than one penetrating keratoplasty. Fifteen optical (A: 4, B: 11) and six tectonic penetrating keratoplasties (A: 4, B: 2) were performed. In seven patients translocation of the mucosal transplant was necessary, four times to remove the mucosa from the initially completely covered cornea. In four patients a second mucosal transplant was performed because the initial graft proved to be insufficient in size to support adequate production of mucus. Most of the additional surgical procedures described were performed to improve visual acuity of the patients. Ten surgical procedures were necessary to correct lid

Table 3 Additional surgical procedures after autologous nasal mucosal transplantation

\begin{tabular}{llll}
\hline Surgical procedure & Total & Group & Group B \\
\hline Penetrating keratoplasty & 21 & 8 & 13 \\
Lamellar keratoplasty & 16 & 10 & 6 \\
Removal of eyelashes & 15 & 10 & 5 \\
Tarsorrhaphy & 13 & 7 & 6 \\
Lid procedures & 10 & 7 & 3 \\
Re-symblepharolysis & 8 & 5 & 3 \\
Nasal mucosal translocation & 7 & 7 & - \\
Cataract extraction & 4 & 2 & 2 \\
Re-nasal mucosal transplantation & 4 & 3 & 1 \\
Buccal mucosal transplantation & 3 & 2 & 1 \\
Cyclocryocoagulation & 2 & 2 & - \\
Enucleation & 3 & 3 & - \\
Conjunctival flap & 1 & 1 & - \\
\hline
\end{tabular}




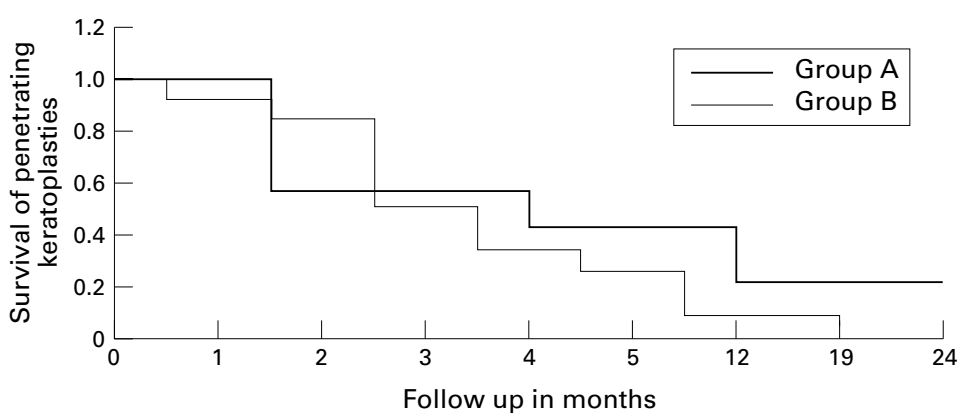

Figure 5 Survival of penetrating keratoplasties after autologous nasal mucosal transplantation (log rank, $p=0.1498$ ).

anomalies and seven to achieve further improvement of ocular wetting. Three eyes had to be enucleated due to painful phthisis and massive scleral necrosis after lye burns.

\section{TRANSPLANT SURVIVAL AFTER PENETRATING} KERATOPLASTIES

There were six tectonic penetrating keratoplasties with five patients developing transplant neovascularisation and corneal clouding within 5 months after surgery. Optical keratoplasties were performed in 13 eyes of eight patients at least 1 year following nasal mucosal grafting. In the majority of patients a lamellar keratoplasty was initially performed to stabilise the ocular surface, to eliminate superficial corneal vascularisation, and to provide the cornea with an intact Bowman's layer for improved fixation of a penetrating keratoplasty. In group A, three of four corneal transplants remained transparent during the follow up period (Fig 2B), but only one cornea in group B (1/12) remained clear until the end of follow up. The remaining grafts resulted in corneal vascularisation or graft failure. The calculated probability of corneal transplant survival after 24 months was $21 \%$ for group A and zero for group B (log rank, $\mathrm{p}=0.1498)($ Fig 5).

VISUAL ACUITY

Final visual acuity ranged from no light perception to $20 / 20$ vision. In comparison with presurgical conditions best corrected visual acuity at the end of follow up was increased in 23 eyes (A: 17/38, B: 6/17), decreased in 11 eyes (A: $7 / 38, B: 4 / 17$ ), and remained unchanged in 21 eyes (A: 14/38, B: 7/17) (Fig 6). Ten patients $(18.2 \%)$ reached a final visual acuity equal to or greater than $20 / 200$. Only one of these patients belonged to group B. In group A, final visual acuity was limited as a result of glaucoma in five patients, a macular hole in one patient, and following enucleation after painful phthisis or massive scleral necrosis in three patients (Tables 1 and 2). The mean outcome in visual acuity was better in group A than in group $\mathrm{B}(\mathrm{p}=0.043, t$ test $)$.

\section{PATIENTS' COMPLAINTS}

Complaints before transplantation like pain and dry eye symptoms improved markedly in 44 patients who experienced presurgical symptoms (44/47). Three patients (6\%) with mainly moderate preoperative symptoms did not report any changes. No patient complained

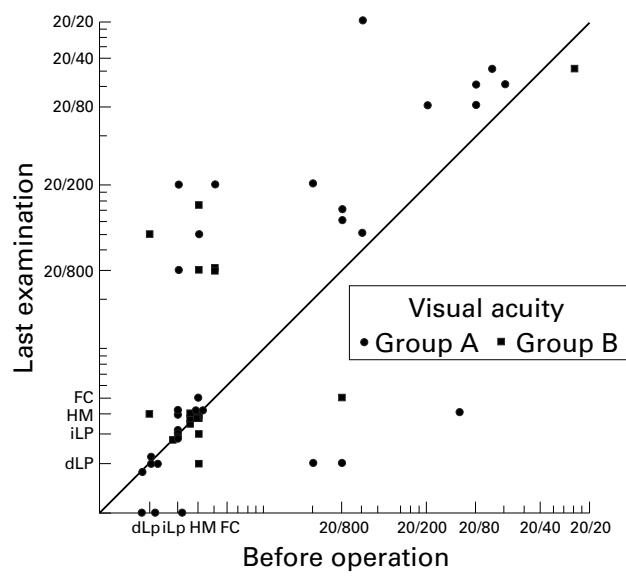

Figure 6 Visual acuity in patients before nasal mucosal transplantation and at last examination. $d L P=$ light perception without projection; $i L P=$ light perception with projection; $H M=$ hand movement $F C=$ finger counting

about continuous pronounced pain or about aesthetic disfigurement after nasal mucosal transplantation. No differences in subjective symptoms were noticed between groups.

SCHIRMER TESTS

Schirmer tests before and after transplantation were available from 26 patients. Presurgical Schirmer test results ranged from 0 to $14 \mathrm{~mm}$ (average $3.0 \mathrm{~mm}$ ); tests at last follow up examination after transplantation ranged from 3 to $30 \mathrm{~mm}$ (average $12.2 \mathrm{~mm}$ ). Tear measurements increased in 23 eyes, decreased in two eyes, and remained unchanged in one eye. A statistically significant increase of Schirmer test results was determined after grafting calculated by Wilcoxon matched pairs test $(\mathrm{p}<0.0001)$. Comparing the two different patient groups there was a statistically significant increase in Schirmer test results $(\mathrm{p}=0.0005)$ in group A $(n=18)$. In group $B(n=8)$ an improvement was observed but was not statistically significant $(\mathrm{p}=0.0759)$, showing an increase in six eyes, a decrease and no changes respectively in one eye.

HISTOLOGY AND IMPRESSION CYTOLOGY

Biopsies of all nasal mucosal grafts of patients from groups A and B were obtained during transplantation. Examined by light microscopy all specimens showed intact nasal mucosa with large numbers of mucin producing goblet cells. All biopsies of nasal mucosal grafts after transplantation showed a great number of mucin producing goblet cells on the surface of the epithelium and in the subepithelial tissue. Biopsies were obtained from 17 patients at several intervals after transplantation (mean 4.6 years). Specimens taken up to 10 years after grafting still revealed numerous goblet cells (Figs 1 and 7). The density of intraepithelial goblet cells ranged from 10 to 39 cells/field with $400 \times$ magnification (median 25 goblet cells/field). An excellent non-invasive procedure to study epithelial changes and the number of persisting goblet cells is impression cytology (Fig 4). The number of goblet cells before and after mucosal transplantation was determined in 22 patients showing an increase 


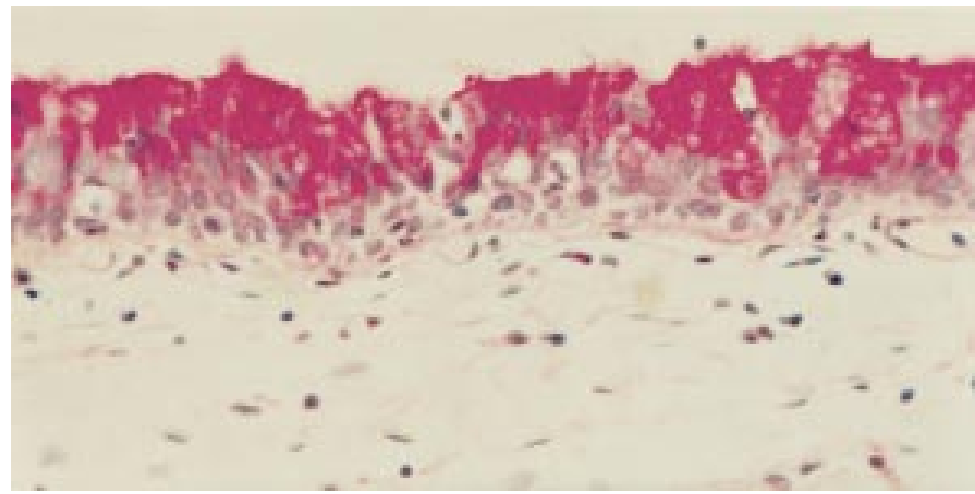

Figure 7 Histological appearance of transplanted autologous nasal mucosa with numerous intraepithelial mucin producing goblet cells. Biopsy taken 5 years after mucosal transplantation (periodic acid-Schiff stain, original magnification $\times 400$ ).

in all eyes. No statistical difference was observed between group A and B. The mean density of goblet cells before nasal mucosal grafting was $48 / \mathrm{mm}^{2}$ and after transplantation $432 / \mathrm{mm}^{2}$ ( $\mathrm{p}<0.0001$; Wilcoxon matched pairs test). No morphological differences in the epithelium were seen by impression cytology or by histology between specimens in groups A and B.

\section{Discussion}

Patients with severe mucus deficiency syndromes suffer from multiple problems including symblepharon, intense pain, reduced visual acuity, corneal vascular scarring, and lid disorders. ${ }^{15} 16$ Treatment often requires multiple operations and frequently the final outcome is not completely satisfactory. The difficulties in treating these patients are clearly visible in the great variety of different approaches and treatment modalities. ${ }^{16}$ The major aim of treatment is to re-establish the ocular surface, separate symblepharon to allow bulbar motility, ensure adequate wetting of the eye, and prevent further complications due to scarring and chronic inflammation. To achieve this goal a mucosal transplant is frequently needed in patients with severe mucus deficiency syndromes to re-create the ocular surface and restore the mucin component of the tear film. This procedure may be followed by optical rehabilitation including keratoplasty and, eventually, transplantation of limbal stem cells. Optical rehabilitation can only be achieved after the ocular surface has been re-established and ocular wetting is sufficient to support a corneal graft.

Different tissues have been advocated to re-establish the ocular surface. Autologous conjunctival grafts from the fellow eye are the best way to restore destroyed conjunctiva. ${ }^{1-3}$ Unfortunately, this is not feasible in all patients, primarily because many disorders involve both eyes. In our study about $60 \%$ of the patients had bilateral involvement. Also, in severe ocular burns, with involvement of the entire conjunctiva and cornea, reconstruction of the fornix and substitution of the ocular surfaces may often demand more mucosa than one can safely excise from the fellow eye.

When autologous conjunctiva is not available, allogeneic conjunctiva from a donor cadaver eye or from a living relative may be used as a substitute. Though providing limbal stem cells to resurface the corneal epithelium, this procedure is not always suitable for the reconstruction of a severely compromised ocular surface. Despite some success, ${ }^{7718}$ systemic immunosuppression is often required and there is a high risk of transplant rejection. This is especially true in irritated and inflamed eyes with mucus deficiency. Eventually, conjunctival graft rejection may also have a negative influence on the outcome of penetrating keratoplasties in these high risk patients. ${ }^{19}$ At least for allogeneic grafts from living relatives there is also a limit to the amount of tissue available.

Since conjunctival tissue maybe unavailable for transplantation, many authors are using other autologous mucosa - for example, buccal or lip mucosa ${ }^{68}$ hard palate mucosa, ${ }^{9}$ maxillary sinus mucosa, ${ }^{10}$ or more recently amniotic membrane. ${ }^{11} 12$ Buccal mucosa was used with promising results to rehabilitate the eyelids of patients with ocular cicatricial pemphigoid. ${ }^{8}$ However, buccal mucosa lacks intraepithelial goblet cells, which are probably important for ocular wetting. Buccal mucosa only carries subepithelial mucosal glands, which are lost during surgical preparation and transplantation and there seems to be a tendency of shrinkage after transplantation. ${ }^{1}$ Mucosa derived from the maxillary sinus is most difficult to obtain, ${ }^{10}$ and hard palate mucosa is thicker than the other types of mucosa and therefore not an ideal substitute for bulbar conjunctiva. ${ }^{9}$ Nowadays, amniotic membrane is also being used to promote conjunctival epithelialisation and to prevent excessive subconjunctival fibrosis. ${ }^{11}$

In our study we used free, autologous, nasal mucosal grafts for several reasons: (1) Nasal mucosal transplantation is feasible in patients with bilateral conjunctival disease as long as intact nasal mucosa exists. (2) The favourable density of intraepithelial goblet cells allows for the preparation of a very thin mucosa, which reduces the risk of postoperative shrinkage of transplanted tissue. (3) The tissue needed is available in relatively large amounts at low risk to the patient. Thus, great areas of denuded sclera can be covered and conjunctival fornices can be reconstructed. If necessary, a second transplantation can make use of a nasal turbinate from the other side of the nose. (4) In appropriate cases additional limbal stem cell transplantation is possible after nasal mucosal grafting has improved the conjunctival disorder. (5) No systemic immunosuppression is required.

In our series, we were able to show vital goblet cells persisting in autologous nasal mucosal grafts for up to 10 years after transplantation. The grafts easily healed to the ocular tissues and continuously produced mucin. This could be seen clinically, and be confirmed by histology and impression cytology. No mucosal graft failed and no serious intraoperative complications did occur. Apart from increased mucin production after nasal mucosal grafting there was a marked improvement of Schirmer 
test results measuring only the watery part of the tear film. Improved Schirmer results may be the consequence of the reconstruction of the ocular fornices, with better distribution of aqueous tears.

Transplanted nasal mucosa is derived from the respiratory tract and may produce mucins differing from that of the ocular surface. However, in normal conjunctiva mucins I and IV are produced by epithelial cells and mucin $\mathrm{V}_{\mathrm{AC}}$ by goblet cells, ${ }^{20}$ all of which are also identified in respiratory epithelium..$^{20}$ Further investigations of mucin production by normal conjunctiva and transplanted mucosa and of their functional importance for ocular wetting are of future interest.

Transplantation of free, autologous, nasal mucosal grafts seems to be an appropriate method to re-establish the ocular surface and to facilitate ocular wetting. In the majority of patients $(90 \%)$ in both groups (A and B) subjective complaints improved significantly. None of the patients reported continuous pronounced pain and circumscribed recurrent symblepharon occurred in only $23 \%$ of patients without restriction of eye movement. Interestingly, the morphological evaluation by impression cytology and histology revealed no differences between the specimens of group and $\mathrm{A}$ and B. Possibly, nasal mucosa may have been minimally affected in the systemic mucosal disease. However, the vital intraepithelial goblet cells kept producing mucin after transplantation to the ocular surface. In contrast, the functional outcome was reduced in group B, with a higher frequency of recurrent symblepharon $(p=0.047)$ and a lower mean visual acuity at final examination compared with group A $(p=0.043)$. Schirmer test results also showed only a non-significant increase in group B. These results are most probably not only due to a potential impairment of nasal mucosa in systemic mucosal disease but also related to the more complicated initial situation in these patients, with more severe symblepharon and a longer lasting mucus deficiency. Nevertheless, there was a reasonable benefit for both groups of patients suggesting that nasal mucosal transplantation is beneficial for traumatic disease as well as for systemic mucosal disease (as long as intact nasal mucosa is available).

Following rehabilitation and better wetting of the ocular surfaces, optical rehabilitation of the patients was the next aim. Here, the long lasting problems of corneal transplantation in high risk patients with heavily vascularised corneas and lid anomalies limited our postoperative results. Only one out of six tectonic and four out of 13 optical penetrating keratoplasties remained transparent during the follow up period. Additionally, known intraocular changes after severe ocular burns like glaucoma, endophthalmitis, or loss of anterior chamber after corneal melting ${ }^{21}$ reduced the final visual acuity in at least nine patients of group A (Table 1). The estimated probability of transplant survival after 24 months was extremely low in group B (Fig 5).
Autologous nasal mucosa is not able to supply the eye with limbal stem cells, which might be required in some of our patients to supply normal corneal epithelium and to support a better survival of corneal transplants. Transplantation of autologous conjunctiva including limbal stem cells or transplantation of corneal grafts including limbal stem cells might serve this purpose, and is planned in some of our patients.

The main therapeutic aim of mucosal transplantation in severe mucus deficiency is the stabilisation of the ocular surfaces and the relief of pronounced pain. Nasal mucosal grafting remains a helpful surgical tool to achieve this effect in patients with severe mucus deficiency syndromes (after ocular burns and in systemic mucosal disease). Nasal mucosal grafts are able to re-establish the mucus components of the tear film, improve symptoms of patients, facilitate wetting of the ocular surface, and open up a potential for visual improvement after keratoplasty.

1 Vastine DW, Stewart WB, Schwab IR. Reconstruction of the periocular mucous membrane by autologous transplantation. Ophthalmology 1982;89:1072-81.

2 Thoft RA. Conjunctival transplantation. Arch Ophthalmol 1977;95:1425-7.

3 Kenyon KR, Tseng SCG. Limbal autograft transplantation for ocular surface disorders. Ophthalmology 1989;96:70923

4 Ballen PH. Mucous membrane grafts in chemical (lye) burns. Am f Ophthalmol 1963;55:302-12.

5 Thies O. Bindehautplastik bei schweren Verätzungen in der chemischen Industrie. Arch f Ophthal 1925;115:246-59.

6 Dening R. Transplantation von Mundschleimhaut bei verschiedenen Erkrankungen der Hornhaut und bei Verbrennungen und Verätzungen des Auges. Arch f Ophthal 1927;118:4729-37.

7 Tsubota K, Satake Y, Ohyama M, et al. Surgical reconstruction of the ocular surface in advanced ocular cicatricial pemphigoid and Stevens-Johnson syndrome. Am f Ophthalmol 1996;122:38-52.

8 Shore JW, Foster CS, Westfall CT, et al. Results of buccal mucosal grafting for patients with medically controlled ocular pemphigoid. Ophthalmology 1992;99:383-95.

9 Manner GE, Mathers WD, Wolfley DE, et al. Hard-palate mucosa graft in Stevens-Johnson syndrome. Am f Ophthalmol 1994;118:786-91.

10 Fry TL, Wood CI. Readily available full-thickness mucous membrane graft. Arch Otolaryngol Head Neck Surg 1987; 113:770-1.

11 Shimazaki J, Yang HY, Tsubota K. Amniotic membrane transplantation for ocular surface reconstruction in patients with chemical and thermal burns. Ophthalmology 1997;104:2068-76.

12 Tsubota K, Satake Y, Ohyama M, et al. Surgical reconstruction of the ocular surface in advanced ocular cicatricial
pemphigoid and Stevens-Johnson syndrome. Am $\mathcal{F} O p h-$ thalmol 1996;122:38-52.

13 Naumann GOH, Lang GK, Rummelt V, et al. Autologous nasal mucosa transplantation in severe bilateral conjunctival mucus deficiency syndrome. Ophthalmology 1990;97: 1011-17.

14 Wenkel H, Rummelt V, Naumann GOH. Autologe Nasenschleimhauttransplantation frühzeitig nach schwersten okularen Verätzungen. Ophthalmologe 1997;94:104-8.

15 Wagoner MD, Kenyon KR. Chemical injuries to the eye. In: Albert DM, Jakobiec FA, eds. Principles and practice of ophthalmology. Vol 5. Philadelphia: WB Saunders, 1994:234-5.

16 Chiou AG, Florakis GJ, Kazim M. Management of conjunctival cicatrizing diseases and severe ocular surface dysfunction. Surv Ophthalmol 1998;43:19-46.

17 Tsai RJ-F, Tseng SCG. Human allograft limbal transplantation for corneal surface reconstraction. Cornea 1994;13: 389-400.

18 Kwitko S, Mainho D, Barcaro S, et al. Allograft conjunctival transplantation for bilateral ocular surface disorders. Ophthalmology 1995;102:1020-5.

19 Thoft RA, Sugar J. Graft failure in keratoepithelioplasty. Cornea 1993;12:362-5.

20 Inatomi T, Spurr-Michaud S, Tisdale AS, et al. Expression of secretory mucin genes by human conjunctival epithelia. Invest Ophthalmol Vis Sci 1996;37:1684-92.

21 Kuckelkorn R, Kottek A, Reim M. Intraokulare Komplikationen nach schweren Verätzungen- Häufigkeit und chirurgische Behandlung. Klin Monatsbl Augenheilkd 1994;205: 86-92. 